\title{
The solution of the reference hypernetted chain equation for the dipolar hard diatomic fluid
}

Cite as: J. Chem. Phys. 91, 2581 (1989); https://doi.org/10.1063/1.457018

Submitted: 03 February 1989. Accepted: 24 April 1989 . Published Online: 31 August 1998

E. Lomba, M. Lombardero, and J. L. F. Abascal

\section{ARTICLES YOU MAY BE INTERESTED IN}

Hypernetted chain solutions for the classical one-component plasma up to $\Gamma=7000$ The Journal of Chemical Physics 61, 2680 (1974); https://doi.org/10.1063/1.1682399

The solution of the hypernetted-chain approximation for fluids of nonspherical particles. A general method with application to dipolar hard spheres

The Journal of Chemical Physics 82, 429 (1985); https://doi.org/10.1063/1.448764

A general solution of the molecular Ornstein-Zernike equation for spheres with anisotropic adhesion and electric multipoles

The Journal of Chemical Physics 92, 3741 (1990); https://doi.org/10.1063/1.457832
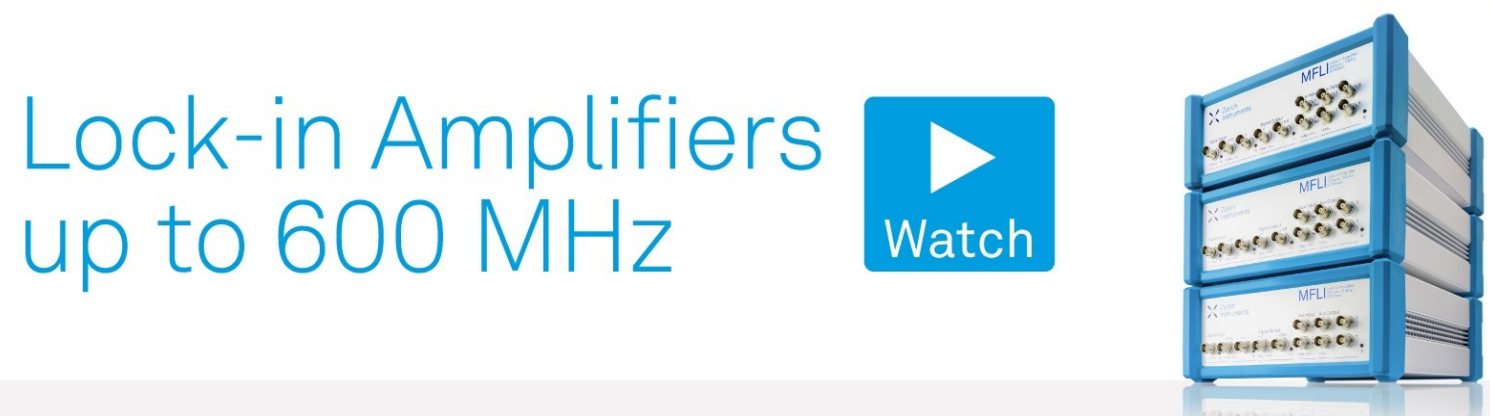


\title{
The solution of the reference hypernetted chain equation for the dipolar hard diatomic fluid
}

\author{
E. Lomba and $M$. Lombardero \\ Instituto de Química Fisica Rocasolano, CSIC, Serrano 119, E-28006 Madrid, Spain \\ and Departamento Química Fisica, Facultad de CC. Quimicas, Universidad Complutense de Madrid, E- \\ 28040 Madrid, Spain
}

J. L. F. Abascal

Departamento Química Fisica, Facultad de CC. Químicas, Universidad Complutense de Madrid, E-28040 Madrid, Spain

(Received 3 February 1989; accepted 24 April 1989)

The reference hypernetted chain equation (RHNC) for a fluid of dipolar hard diatomics was solved numerically. Three choices for the reference bridge function $B_{0}(12)$, were examined. The simplest, $B_{0}(12)=0(\mathrm{HNC})$ and $B_{0}(12)$ corresponding to the uncharged homonuclear hard diatomic fluid, computed from simulation data and by the Percus-Yevick approximation. The computed fluid structure [i.e., the $g(12)$ expansion coefficients] showed a remarkable quantitative agreement with the structure obtained from a mean reaction field Monte Carlo simulation. The same applied to the configurational energy. The values for the dielectric constant, however, furnished only qualitatively indications of the density dependence of this quantity. The probable origin of this discrepancy is analyzed.

\section{INTRODUCTION}

It has been firmly established that the influence of molecule shape on the structure and macroscopic properties of dipolar fluids must not be overlooked. ${ }^{1-3}$ However, to date very little progress has been achieved in applying theoretical methods to the study of fluids composed of nonspherical particles carrying high dipole moments, even though in recent years integral equation methods (in particular the reference hypernetted chain equation ${ }^{4-7}$ ) have proved to be very successful tools in determining the structure and dielectric properties of polar fluids consisting of spherically shaped particles. To our knowledge, dipolar fluids of anisotropic particles have been considered mostly in the context of the site-site Orstein-Zernike (OZ) equation ${ }^{8}$ using mean spherical approximation-like (MSA) and hypernetted chain-like (HNC) closures. ${ }^{9,10}$ Although such site-site approaches may offer advantages in certain cases (e.g., comparison with experimental atom-atom structure factors), procedures furnishing the molecular pair distribution function $g(12)$ are generally preferable, since this latter function contains more information about the system than its site-site counterparts. In this respect, some attempts have been made to apply perturbation theories that yield the full $g(12)$ function to dipolar molecular fluids. Steinhauser and Bertagnolli ${ }^{11}$ used a perturbation treatment with a nonspherical reference system in conjunction with the Percus-Yevick (PY) approximation. Their results showed sizeable deviations for some of the expansion coefficients of $g(12)$ (as compared to experimental data), although the computed values for the dielectric constant appeared to agree quite well with the experimental results. An important drawback attaching to this sort of theory lies in the complexity of the reference system, whose properties tend to be unavailable.

The use of the RHNC equation, which, as just mentioned above, proved quite successful for dipolar systems of spherically shaped particles, is an attractive alternative to perturbation techniques. In this paper, we report on solutions to the RHNC equation for a model of dipolar fluids previously studied by us using a mean reaction field Monte Carlo simulation. ${ }^{3}$ We have investigated different choices for the bridge function, $B(12)$, in the RHNC equation, ${ }^{4}$ namely $B(12)=0$ [which reduces to the hypernetted chain approximation (HNC)] and $B(12)=B_{0}(12), B_{0}(12)$ being the bridge function for the reference system, an uncharged hard diatomic with the same shape as the dipolar molecule; the corresponding spherical harmonic coefficients of the function $B_{0}(12)$ were determined by the authors from MC data and the PY aproximation. ${ }^{12}$ These two approaches will be hereinafter referred to as RHNC (MC) and RHNC (PY), respectively.

Section II describes the model for the polar fluid for which computations were performed, and sets out the chief expressions relating microscopic structure [i.e., the pair distribution function, $g(12)$, or its spherical harmonic coefficients] to macroscopic properties. For the sake of completeness, Sec. II B summarizes the algorithm used to solve the RHNC equation, which has been fully described elsewhere. ${ }^{4,5,7}$ Section III considers the numerical results for the microscopic structure (spherical harmonic coefficients), configurational energy, and dielectric properties.

\section{SUMMARY OF THE THEORY}

In this section we first examine the explicit expression of the interaction potential for our polar fluid model and the relations for thermodynamic and dielectric properties which can subsequently be derived. We will also briefly summarize the main points concerning solution of the RHNC equation for a fluid made up of linear molecules. 


\section{A. A theoretical model for dipolar diatomic fluids}

The chief characteristics of a dipolar molecular liquid are, clearly, molecular anisotropy and dipole moment. The simplest system capable of modeling such a fluid is doubtless the dipolar homonuclear hard diatomic. Earlier we investigated this model in some detail ${ }^{3}$ by means of a Monte Carlo simulation with the mean reaction field technique to handle the long-range interactions. In order to remain consistent with our previous computations, we have again used here the same set of molecular parameters set out in our previous paper. ${ }^{3}$ The MC data can thus be used as benchmark results for testing our theoretical predictions. Consequently, the dipolar potential must include the reaction field term, and hence the full pair potential is expressed

$$
u(12)=u_{\mathrm{HD}}(12)+u^{\mathrm{MRF}}(12)
$$

with

$$
u^{\mathrm{MRF}}(12)=\left\{\begin{array}{rr}
-\frac{\mu^{2}}{r^{3}} \Phi^{112}(12)-\frac{2\left(\epsilon^{\prime}-1\right)}{2 \epsilon^{\prime}+1} & \frac{\mu^{2}}{R_{c}} \Phi^{110} \\
0 & \text { if } r \leqslant R_{c} \\
0 & \text { if } r>R_{c}
\end{array}\right.
$$

where $u_{\mathrm{HD}}(12)$ denotes the pair potential of an uncharged hard diatomic (in this case, two fused hard spheres of diameter $\sigma$ and a separation between centers $l=0.6 \sigma$ ), $\mu$ is the dipole moment, $R_{c}$ is the cutoff radius and $\epsilon^{\prime}$ is the dielectric constant outside the truncation sphere. The last two parameters are only meaningful in the context of the mean reaction field approach. We have set $\epsilon^{\prime}$ to 50 and $R_{c}$ to $4 \sigma$, for consistency with the MC calculations. The quantities $\Phi^{112}(12)$ and $\Phi^{110}(12)$ are rotational invariants that can be expressed in terms of the molecular orientations as

$$
\begin{aligned}
& \Phi^{110}(12)=s_{1} \cdot s_{2} \\
& \Phi^{112}(12)=3\left(s_{1} \cdot r\right)\left(s_{2} \cdot r\right) / r^{2}-\left(s_{1} \cdot s_{2}\right),
\end{aligned}
$$

where $s_{i}$ is a unit vector that describes the dipole orientation of particle $i$ and $\mathbf{r}$ is the vector joining the centers of molecules 1 and 2.

Within this choice of interaction potential, the configurational energy of the dipolar fluid can be written

$$
\begin{aligned}
\beta u_{D D}= & -3 y \int_{0}^{R_{c}} h^{112}(r) r^{-1} d r \\
& -\frac{3}{2} y \frac{2\left(\epsilon^{\prime}-1\right)}{2 \epsilon^{\prime}+1} \int_{0}^{R_{c}} h^{110}(r) r^{2} d r
\end{aligned}
$$

where $y=4 \pi \rho \mu^{2} / 9 k T \sigma^{3}$ and $h^{110}(r)$ and $h^{112}(r)$ are

$$
\begin{aligned}
& h^{110}(r)=g_{110}(r)-2 g_{111}(r), \\
& h^{112}(r)=g_{110}(r)+g_{111}(r),
\end{aligned}
$$

the functions $g_{k i m}(r)$ being the coefficients of the spherical harmonic expansion of the molecular pair distribution function. The Kirkwood relation for the dielectric constant ${ }^{13}$ was also modified to yield the following expression ${ }^{14}$ :

$$
\begin{aligned}
& \frac{(\epsilon-1)\left(2 \epsilon^{\prime}+1\right)}{3\left(\epsilon+2 \epsilon^{\prime}\right)}=y g_{k}, \\
& g_{k}=1+\frac{1}{3} \rho \tilde{h}^{110}(0) \\
& =1+\frac{4 \pi}{3} \rho \int_{0}^{\infty} h^{110}(r) r^{2} d r .
\end{aligned}
$$

In the above expression the tilde denotes a Fourier transform, and $g_{k}$ is the Kirkwood $g$ factor, obtainable from simulation as $g_{k}=\left\langle\mathbf{M}^{2}\right\rangle / N \mu^{2}$, where $\mathbf{M}=\Sigma \boldsymbol{\mu}_{i}$.

It is customary to define a reduced squared dipole moment $\mu^{*^{2}}=4 \pi \beta \mu^{2} / \sigma^{3}$, a quantity that in our model has been set to $\mu^{*^{2}}=2.452$ (which implies a high dipole strength). The density dependence of the results was investigated by performing calculations at several densities $\left(\rho^{*}=\rho \sigma^{3}\right)$, namely $\rho^{*}=0.2,0.3,0.4352$, and 0.5 (this last case was computed only for the HNC approximation). It should be mentioned here that, while the use of point dipoles for dipolar molecular fluids is unusual in simulations (although in Ref. 3 it was shown that such models accounted quite well for the most interesting features of dipolar fluids), Steinhauser $e t a l .{ }^{11}$ also employed a point dipole potential to determine theoretically the $g(12)$ expansion for a molecular polar fluid.

\section{B. Solution of the RHNC equation}

The RHNC equation for linear fluids is

$$
\begin{aligned}
S(12)= & \frac{\rho}{4 \pi} \int c(13)[S(32)+c(32)] d \omega_{3} d \mathbf{r}_{3}, \\
c(12)= & \exp \left[-\beta u(12)+S(12)-B_{0}(12)\right] \\
& -1-S(12),
\end{aligned}
$$

where Eq. (7) is the Orstein-Zernike $(\mathrm{OZ})$ relation for $S=h-c$ and Eq. (8) is the RHNC closure, in which $B_{0}(12)$ is the bridge function for the reference system. Note that Eq. (8) differs from the RHNC closure presented in Refs. 4, 5, and 7 in the sign of the bridge function. The special form of Eq. (8) was chosen for consistency with the bridge function previously computed by the authors ${ }^{12}$ and is the same as that set out in several other papers (e.g., Ref. 15). Various choices are available for the function $B_{0}(12)$ : it may either be set to zero (HNC equation) or the values for any given reference system may be used. For this latter instance we considered the natural reference fluid to be the uncharged hard diatomic. The values for this $B_{0}(12)$ were obtained earlier by the authors ${ }^{15}$ by means of MC simulation and the Percus-Yevick approximation. Use of a form of $B_{0}(12)$ in the PY approximation for a hard diatomic in Eq. (8) renders a PY closure relation only at the limit of vanishing dipole strength, and therefore with this choice of $B_{0}(12)$ the long-range behavior of the fluid can be expected to be taken adequately into account by Eq. (8). Direct use of the PY closure would not be sensible for long-range potentials. In a similar context, Lado $^{5(b)}$ employed a $B_{0}(12)$ for a hard diatomic fluid in the PY approximation as the reference function for a two center Lennard-Jones ( $\mathrm{LJ}$ ) fluid, with excellent results. Optimization of the reference-hard-di- 
atomic diameter $\sigma$, which considerably improved the results for Lennard-Jones ( $\mathrm{LJ}$ ) fluid, is extremely time consuming yet does not play a crucial role when only dipolar potentials are involved ${ }^{7}$; therefore, the nonoptimized version of the theory only was used in the present instance.

The pair functions appearing in Eqs. (7) and (8) must be expanded in spherical harmonics as

$$
S(12)=4 \pi \sum_{k l m} S_{k l m}\left(r_{12}\right) Y_{k m}\left(\omega_{1}\right) Y_{1-m}\left(\omega_{2}\right)
$$

and similarly for the functions $c(12)$ and $B_{0}(12)$. Equation (7) must be solved in Fourier space, becoming

$$
\begin{aligned}
\widetilde{S}\left(\mathbf{k}, \omega_{1}, m \omega_{2}\right)= & \frac{\rho}{4 \pi} \int \tilde{c}\left(\mathbf{k}, \omega_{1}, \omega_{3}\right) \\
& \times\left[\tilde{S}\left(\mathbf{k}, \omega_{1}, \omega_{3}\right)+\tilde{c}\left(\mathbf{k}, \omega_{1}, \omega_{3}\right)\right] d \omega_{3}
\end{aligned}
$$

Using expansions similar to those given in Eq. (9) (but with a rotated reference frame) in Fourier space in Eq. (10) while taking into account the orthogonality of the spherical harmonics, yields the following set of matrix equations

$\widetilde{\mathbf{S}}_{m}(k)=(-1)^{m} \rho\left[\mathbf{I}-(-1)^{m} \rho \tilde{\mathbf{c}}_{m}(k)\right]^{-1}\left[\tilde{\mathbf{c}}_{m}(k)\right]^{2}$,

where $I$ is the identity matrix. The elements of the $\widetilde{\mathbf{S}}_{m}$ and $\tilde{\mathbf{c}}_{m}$ matrices are $\widetilde{S}_{k l m}(k)$ and $\tilde{c}_{k l m}(t)$, respectively. Simultaneously, the closure relation (8) can be rewritten as

$$
\begin{aligned}
c_{k l m}\left(r_{12}\right)= & \left\langle g\left(r_{12}, \omega_{1}, \omega_{2}\right) \mid k l m\right\rangle \\
& +\delta_{k l m, 000}-S_{k l m}\left(r_{12}\right)
\end{aligned}
$$

with

$$
\begin{aligned}
g\left(r_{12}, \omega_{1}, \omega_{2}\right)= & \exp \left\{-\beta u(12)+4 \pi \sum_{\mu \nu \lambda}\left[S_{\mu \nu \lambda}\left(r_{12}\right)\right.\right. \\
& \left.\left.-B_{0_{\mu \nu \lambda}}\left(r_{12}\right)\right] Y_{\mu \lambda}\left(\omega_{1}\right) Y_{\nu-\lambda}\left(\omega_{2}\right)\right\}
\end{aligned}
$$

and $\langle\ldots \mid k l m\rangle$ denotes the projection onto the product $Y_{k m}\left(\omega_{1}\right) Y_{1-m}\left(\omega_{2}\right)$.

Thus, once an initial solution [a set of $S_{k l m}(r)$ ] is available, the $c_{k l m}(r)$ can be computed by using Eq. (12). After some mathematical manipulation these functions yield the set of $\tilde{c}_{k l m}(k)$. Note that $\tilde{c}_{k l m}(k)$ are not the Fourier transforms of $c_{k l m}(r)$, and consequently the procedure implies a change of reference frame and a Hankel transform. ${ }^{4,6}$ Full details are available in Ref. 4 . Inserting the $\tilde{c}_{k l m}(k)$ coefficients into Eq. (12) gives the set of $\widetilde{S}_{k l m}(k)$, which can be inverted to give a new set of $S_{k l m}(r)$, and a new iteration may proceed from Eq. (11). As indicated in Ref. 4, Gillan's method $^{16}$ for the radial coefficient $S_{000}(r)$, is very convenient to speed up convergence. An important part of this algorithm was implemented in a program published in the literature, ${ }^{17}$ which we modified to account for dipolar potentials.

\section{RESULTS}

The RHNC solutions were obtained using 512 and 1024 grid points at a grid width of $\Delta r=0.02 \sigma$. Unlike the case of dipolar hard spheres, ${ }^{7}$ the results were not very sensitive to the number of grid points. On the other hand, the crux of the computation is the number of points used in the angle averages of Eq. (12). In the region $\left(\sigma^{2}-\frac{1}{2}^{2}\right)^{1 / 2}<r<1+\sigma$, in which the highest degree of anisotropy is to be expected, 30 points per angle in Gaussian integrations ${ }^{5}$ seemed to ensure the required accuracy. When $r>1+\sigma$, the interaction is simply the dipole-dipole potential of Eq. (2), which is a continuous smooth function, and ten points per angle were used in the averaging. For $r<\left(\sigma^{2}-\frac{1}{2}^{2}\right)^{1 / 2}$ the hard core of our model implies $g(12)=0$.

\section{A. Pair correlation function}

The pair correlation function $h(12)=g(12)-1$ was examined in terms of its spherical harmonic coefficients, namely the radial distribution function $g_{000}(r)$ as well as the projections $h^{110}(r)$ and $h^{112}(r)$. These functions are shown in Figs. 1-3 for $\rho^{*}=0.4352$. Discrepancies between simulation and theory are, obviously, less appreciable at lower densities. The various theoretical approaches derived from different choices of $B_{0}(12)$ have been indicated in the figures by different types of lines.

Figures 1-3 show the theoretical results to agree remarkably well with the simulation values. Specifically, the HNC approximation reproduced the MC functions $h^{110}$ and $h^{112}$ almost exactly (Figs. 2 and 3 ). A small deviation can be observed in the positions of the maxima and minima for the coefficient $g_{000}$ (Fig. 1). The RHNC approximation [particularly the RHNC (MC)] slightly improved the HNC, such that the MC values for $g_{000}(r)$ appeared on the RHNC (MC) $g_{000}$ curve (Fig. 1), with the exception of a shoulder occurring in the region $\sigma<r<1+\sigma$. There is a related, though somewhat larger, departure in the $h^{112}$ curve (Fig. 3 ); still, when translated into macroscopic properties by Eq. (4), it turned out to have very little effect. In our opinion, this discrepancy was a spurious effect caused by uncertain-

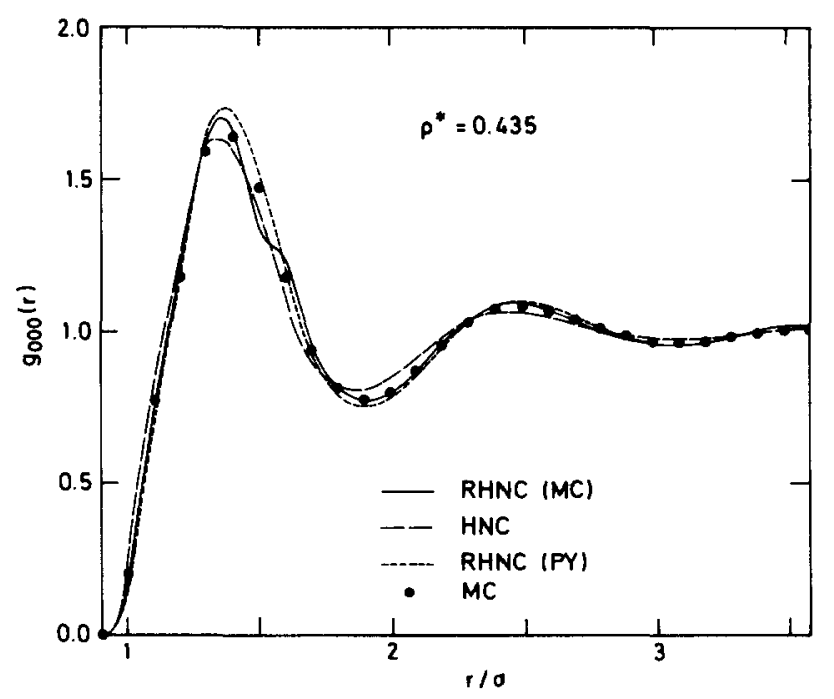

FIG. 1. The radial distribution coefficient $g_{000}$ for $\rho^{*}=0.435$. The lines stand for different choices of $B_{0}(12)$ in the RHNC (see the text) and the dots correspond to simulated data. 


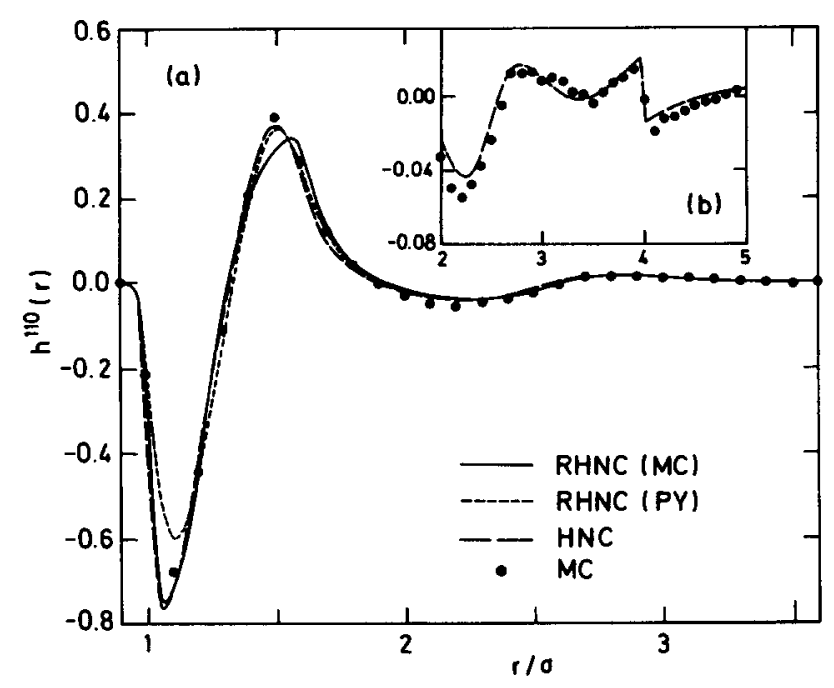

FIG. 2. (a) The projection $h^{110}$ of the total correlation function for $\rho^{*}=0.435$. The symbols have the same meaning as in Fig. 1. (b) A detail of the long-range behavior of $h^{110}$ computed via simulation (dots) and in the HNC approximation (dashed line).

ties in the determination of the bridge function $B_{0}(12)$ from the MC data. ${ }^{12}$

The long-range behavior of the coefficient $h^{110}$ is of special interest, since it makes a major contribution to the dielectric properties. Therefore, the upper right-hand corner of Fig. 2 depicts a detail of the $h^{110}$ function in the range $2 \sigma<r<5 \sigma$, for which MC results were available. The dashed line is the HNC solution, the long-range behavior of which did not differ perceptibly from that computed using the RHNC (MC) or RHNC (PY). It is readily apparent that theory and simulation disagreed only to a very small extent. The discontinuity at $r=R_{c}(4 \sigma)$ occurred in both the $\mathrm{MC}$ and the theoretical results. A similar discontinuity also appeared in the $h^{112}$ coefficient. Both were artifacts of the MRF boundary conditions and would not appear in the case of infinite-range dipolar potentials. Only the discontin-

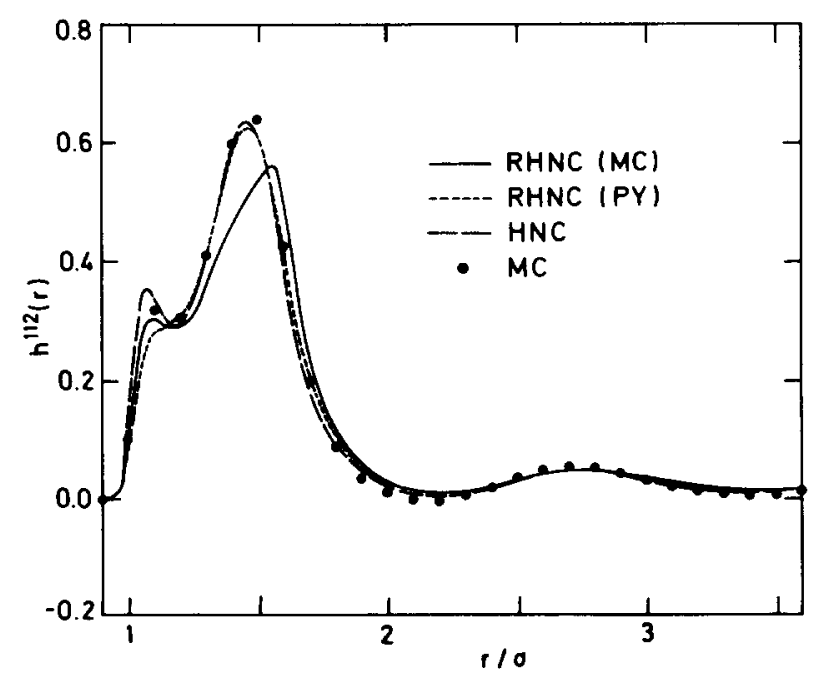

FIG. 3. The projection $h^{112}$ of the total correlation function for $\rho^{*}=0.435$. The symbols have the same meaning as in Fig. 1. uity in function $h^{112}$ has been reported for spherically shaped particles. ${ }^{17,18}$

\section{B. Configurational energy}

Once the coefficients $h^{110}$ and $h^{112}$ have been calculated, the configurational energy can readily be computed from Eq. (4). The results have been summarized in Table I, together with the simulated values from Ref. 3 . The good agreement between theory and simulation was a consequence of the quality of the computed function $h^{112}$ and the fact that Eq. (4) tends to minimize the effect of inaccuracies, especially for large values of $r$. Larger deviations were observable in the RHNC (PY) results, whereas the HNC values reproduced the simulated energies within their statistical uncertainties. The HNC approximation yielded a configurational energy in accordance with the simulated value for the 500 -particle sample, which differed by $2 \%$ from the results for the 256-particle sample. ${ }^{3}$

\section{Dielectric constant and Kirkwood $\boldsymbol{g}$ factor}

The dielectric constant $\epsilon$ can be expressed in terms of the mean squared dipole $\left\langle\mathbf{M}^{2}\right\rangle$ moment of the sample through Eq. (6), where $g_{k}=\left\langle\mathbf{M}^{2}\right\rangle / N \mu^{2}$ is the Kirkwood $g$ factor. The results for $g_{k}$ computed by the RHNC integral equation using Eq. (6b) are set out in Table II. Note that the $\tilde{h}^{110}(0)$ value is readily available in the procedure for solving the integral equation. This avoids integration of $r^{2} h^{110}$ in Eq. (6b), thereby removing a further potential source of error. ${ }^{7}$ Both numerical methods produce the same result if a sufficiently large number of grid points is used (hence the agreement improved when 1024 points were used) as well as at low densities. The values given in Table II were obtained by means of the transforms. The simulated values for $\left\langle\mathbf{M}^{2}\right\rangle_{c} /$ $N \mu^{2}\left(\langle\ldots\rangle_{c}\right.$ denotes that the average has been calculated throughout the cubic simulation cell) from Ref. 3 are also presented in Table II. The theoretical values of $\left\langle M^{2}\right\rangle / N \mu^{2}$ can be seen to differ somewhat from the simulated values, the difference obviously being larger at higher densities. Furthermore, introducing the function $B_{0}(12)$ did not substantially alter the $g$ factors computed, and the RHNC (MC), RHNC (PY), and HNC results were quite similar. Nonetheless, the theoretical values reproduced one of the main features of simulated $g$-factor values in molecular fluids, namely their proximity to the Onsager result $g_{k}=1 .^{1}$ On the other hand, another characteristic, seemingly exhibited only

TABLE I. Comparison between RHNC (MC), RHNC (PY), and HNC results for $\beta\left(U_{D D}\right) / N$ vs the simulated values.

\begin{tabular}{|c|c|c|c|c|}
\hline \multirow[b]{2}{*}{$\rho^{*}$} & \multirow[b]{2}{*}{ MC } & \multicolumn{2}{|c|}{ RHNC } & \multirow[b]{2}{*}{ HNC } \\
\hline & & $B_{k l m}(\mathrm{MC})$ & $B_{k l m}(\mathrm{PY})$ & \\
\hline $\begin{array}{l}0.2 \\
0.3 \\
0.435 \\
0.5\end{array}$ & $\begin{array}{l}-0.582 \pm 5 \times 10^{-4} \\
-0.806 \pm 5 \times 10^{-4} \\
-1.089 \pm 5 \times 10^{-4} \\
-1.233 \pm 5 \times 10^{-4}\end{array}$ & $\begin{array}{c}-0.575 \\
-0.791 \\
-1.054 \\
\cdots\end{array}$ & $\begin{array}{c}-0.573 \\
-0.789 \\
-1.050 \\
\ldots\end{array}$ & $\begin{array}{l}-0.583 \\
-0.807 \\
-1.088 \\
-1.230\end{array}$ \\
\hline
\end{tabular}


TABLE II. Comparison between RHNC (MC), RHNC (PY), and HNC results for $\left\langle\mathbf{M}^{2}\right\rangle / N \mu^{2}$ vs the simulated values.

\begin{tabular}{lcccc}
\hline \hline & \multicolumn{4}{c}{ RHNC } \\
$\rho^{*}$ & MC & $B_{\text {klm }}(\mathrm{MC})$ & $\boldsymbol{B}_{k l m}(\mathrm{PY})$ & $\mathrm{HNC}$ \\
\hline 0.2 & $1.03 \pm 0.03$ & 1.218 & 1.220 & 1.210 \\
0.3 & $1.31 \pm 0.04$ & 1.252 & 1.255 & 1.231 \\
0.435 & $0.85 \pm 0.04$ & 1.259 & 1.281 & 1.225 \\
0.5 & $0.63 \pm 0.03$ & $\cdots$ & $\cdots$ & 1.190 \\
\hline \hline
\end{tabular}

by molecular dipolar fluids, the saturation effect in the $g_{k}$ factor values as density increases (a consequence of the steric hindrance of the free rotation of the molecules), ${ }^{3}$ was qualitatively accounted for. The HNC results (for which values were obtained at $\rho^{*}=0.5$ ) for $g_{k}$ actually decreased in a fashion similar to the MC values. Nevertheless, the theoretical predictions for $g_{k}$ did not match those for the configurational energy or the expansion coefficients. Table III presents the dielectric constant values obtained using the $g$ factors from Table II in Eq. (6a). The aforementioned saturation effect, though actually present in the $g_{k}$ values, was not observable in our theoretical $\epsilon$ values, since it was masked by Eq. (6a). In contrast, the simulated $\epsilon$ values fell at $\rho^{*}=0.4352$, in response to the sharp decrease in $\mathrm{g}_{\mathrm{k}}$, though here, too, the effect was damped somewhat by Eq. (6a).

There are several questions concerning our theoretical results and the relative failure in the computation of $\epsilon$, to which special attention should be addressed. First, it is surprising that the theories employed, which proved quite accurate in determining the microscopic structure and configurational energy, yielded relatively poor results for the dielectric properties. Second, it has been well established that the RHNC theory using a reference bridge function for a hard-sphere system furnished quite accurate results for $\epsilon$ for dipolar hard spheres. ${ }^{8}$ In our case, a similar approach [RHNC (MC)] did not seem to improve the HNC [ $B_{0}(12)$ $=0]$ results at all. These questions are examined below.

In order to provide some information about the sources of error, Fig. 4 depicts the function

$$
g_{k}(R)=1+\frac{4 \pi}{3} \rho \int_{0}^{R} r^{2} h^{110}(r) d r
$$

which is expected to fulfill the limiting condition

$$
\lim _{R \rightarrow \infty} g_{k}(R)=g_{k}=\left\langle\mathbf{M}^{2}\right\rangle / N \mu^{2} .
$$

The lines in Fig. 4 represent the HNC solution at different

TABLE III. Comparison between RHNC (MC), RHNC (PY), and HNC results for $\epsilon$ vs the simulated values.

\begin{tabular}{lcccc}
\hline \hline & \multicolumn{4}{c}{ RHNC } \\
\multicolumn{1}{c}{$\rho^{*}$} & MC & $B_{k l m}(\mathrm{MC})$ & $B_{k i m}(\mathrm{PY})$ & $\mathrm{HNC}$ \\
\hline 0.2 & $3.2 \pm 0.1$ & 3.57 & 3.57 & 3.55 \\
0.3 & $5.0 \pm 0.1$ & 5.01 & 5.02 & 4.95 \\
0.435 & $4.9 \pm 0.2$ & 6.95 & 7.07 & 6.79 \\
0.5 & $4.4 \pm 0.2$ & $\cdots$ & $\cdots$ & 7.51 \\
\hline
\end{tabular}

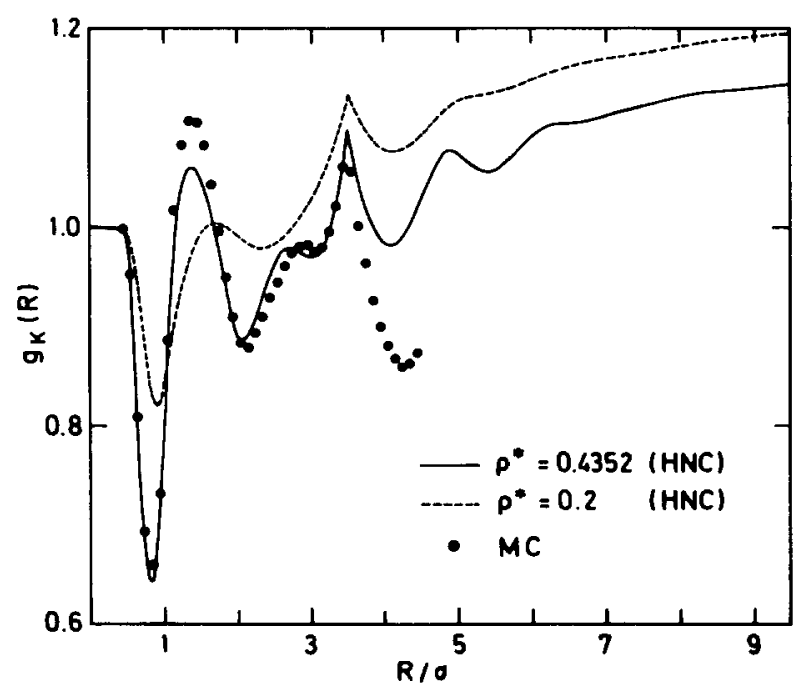

FIG. 4. The $R$-dependent $g$ factor defined by Eq. (13) in the text. The lines stand for the HNC solution at different densities: 0.4352 (solid) and 0.2 (dotted). Solid circles represent the MC data for the highest density.

densities; the dots are the values obtained by integrating the simulated $h^{110}$ functions. For the sake of clarity, only the simulated values for $\rho^{*}=0.4352$ have been shown; the results for lower densities exhibited very similar trends. From the Fig. 4 it may readily be concluded that the HNC equation accurately reproduced the short-range structure of $g_{k}(R)$ (except for a small departure in the first peak) and hence that of the function $h^{110}$, whereas significant deviations occurred for $r>R_{\mathrm{c}}$. These discrepancies must be viewed in the light of the long-range behavior of the function $h^{110}$ depicted in Fig. 2(b). On doing so, it turned out that the very small disagreement between the HNC equation and the simulated function $h^{110}$ gave rise to the large difference observed in $g_{k}(r)$ at $R=5 \sigma$. The upshot of this is that good theoretical prediction of $\left\langle M^{2}\right\rangle / N \mu^{2}$ requires that the tail of the function $h^{110}$ be computed with very high precision. In fact, the contribution of the short-range structure to the $g$ factor is rather small, as can be seen from Fig. 4; hence, small absolute errors in the contribution of the long-range behavior of function $h^{110}$ bring about large relative errors in $\left\langle M^{2}\right\rangle /$ $N \mu^{2}$. This is why very accurate results for the structure and energy can be obtained in conjunction with relatively poor values for $\epsilon$.

The tail of function $h^{110}$ can be regarded as an effect of non-negligible indirect correlations across the cutoff radius and is responsible for the reported differences between $\left\langle M^{2}\right\rangle_{s p h} / N \mu^{2}=g_{k}\left(R_{c}\right)\left(\langle\ldots\rangle_{s p h}\right.$ indicates that the average was performed within the cutoff sphere) and $\left\langle M^{2}\right\rangle_{c} / N \mu^{2}$ $=g_{k} \cdot{ }^{18,19}$ These indirect correlations should be accounted for by the bridge function [ which, by definition, is a multiple convolution of $h(12)^{20}$ ]. It is unlikely that this effect would be adequately accounted for by a $B_{0}(12)$ function, which does not even satisfy the symmetry requirements of the dipole-dipole potential. Note that the hard-core reference fluid is made up of centrosymmetric particles, and this symmetry is broken by the introduction of the dipolar potential. 
This could explain why the HNC values for $\epsilon$ were not basically modified by the introduction of $B_{0}(12)$ in the closure relation: the tail of $h^{110}$ seemingly remained unchanged. However, the function $g_{000}(r)$ was improved somewhat in the RHNC (MC) and RHNC (PY) approaches.

On the other hand, in the case of dipolar hard spheres, $\epsilon$ is also known to be highly dependent on the tail of $h^{110,13}$ but since this function is essentially positive for such models, ${ }^{18,19}$ the contribution of the short- and medium-range structure is probably considerable. Errors in the tail of $h^{110}$ will then have a smaller effect on the accuracy of $\epsilon$. This explains why the RHNC approach achieved far better agreement with simulation for dipolar hard sphere fluids.

Finally dependence on the cutoff radius, another aspect that has been of interest in the application of the MRF method to dipolar fluids, was also examined. Several computed values reproducing those for the infinite range dipolar potential have been reported for dipolar hard spheres. For instance, Patey et al. ${ }^{17,18}$ reported $R_{c}=4.5 \sigma$ whereas Lado et $a l .^{7}$ suggested a value of $R_{c}=6 \sigma$ for higher dipole moments and densities. Our MC computations ${ }^{3}$ did not show any appreciable sensitivity to changes in $R_{c}$. In order to confirm this lack of sensitivity, we solved the HNC equation at $\rho^{*}=0.4352$ using $R_{c}=4 \sigma$ and $6 \sigma$, and the results of these computations are shown in Table IV. The dielectric properties do not seem to be crucially affected by the choice of $R_{c}$ (obviously, further reductions in $R_{c}$ would increase the effect substantially). Moreover, the rise in configurational energy apparent in Table IV is readily explained as a result of the increment in the number of particles interacting with any given molecule as $R_{c}$ augments. It may therefore be concluded that in molecular fluids, in which shape forces are overwhelmingly dominant, the effects of small changes in the range of the dipolar interaction do not introduce substantial changes in the macroscopic quantities.

\section{CONCLUSIONS}

In this paper we have presented the main results obtained by solving the RHNC equation for dipolar hard diatomic fluids using different choices for the reference bridge function. As a general conclusion, the theoretical approaches here examined provided fairly accurate descriptions of the microscopic structure of the fluids, apparently even for the long-range behavior of the pair distribution function. Configurational energy is also correctly predicted by the theory. However, our results for the dielectric properties, though qualitatively correct, differed considerably from the simulated results. Detailed analysis showed the discrepancies to arise from very small deviations in the tail of the function $h^{110}$. This tail stemmed from indirect correlations across the cutoff radius that seemingly are not adequately accounted for when using the function $B_{0}(12)$ corresponding to an uncharged hard-core system. A related situation
TABLE IV. Thermodynamic and dielectric properties computed in the HNC approximation using two different truncation distances $\boldsymbol{R}_{c}$.

\begin{tabular}{cccc}
\hline \hline$R_{c} / \sigma$ & $\beta\left\langle U_{D D}\right\rangle / N$ & $\left\langle M^{2}\right\rangle / N \mu^{2}$ & $\epsilon$ \\
\hline 4.0 & -1.089 & 1.225 & 6.79 \\
6.0 & -1.097 & 1.227 & 6.80 \\
\hline \hline
\end{tabular}

was described by Foiles et al., ${ }^{21}$ who investigated the longrange behavior of $B(12)$ in coulombic fluids in some detail. They reported that the tail of $B(12)$, though small, was important and could not be neglected. They also suggested using of the "real" asymptotic expression for $B(12)$ in combination with the hard-core $B(12)$, which should account for the short-range structure. Their method would appear to be quite promising for improving the results presented herein, but the numerical procedure is sufficiently involved to have prevented, its extension to molecular fluids up to now.

\section{ACKNOWLEDGMENTS}

The authors acknowledge the Dirección General de Investigación Científica y Tecnológica of Spain, which provided financial support for this research under Project SEUI No. PB87-0246-00. They also wish to thank the Computer Centres of CSIC, the Universidad Complutense, and, in particular, CIEMAT for generously contributing the use of their computing facilities.

${ }^{1}$ O. Steinhauser, Mol. Phys. 46, 827 (1982).

${ }^{2}$ G. P. Morriss, Mol. Phys. 47, 833 (1982).

${ }^{3}$ E. Lomba, M. Lombardero, and J. L. F. Abascal, Mol. Phys. (submitted). ${ }^{4}$ F. Lado, Mol. Phys. 47, 283 (1982).

s (a) F. Lado, Mol. Phys. 47, 299 (1982); (b) J. Chem. Phys. 88, 1950 (1988).

${ }^{6}$ P. H. Fries and G. N. Patey, J. Chem. Phys. 82,429 (1985)

${ }^{7}$ F. Lado, M. Lombardero, E. Encisco, S. Lago, and J. L. F. Abascal, J. Chem. Phys. 85, 2916 (1985).

${ }^{8} \mathrm{D}$. Chandler, The Liquid State of Matter, edited by E. W. Montroll and J.

L. Lebowitz (North-Holland, Amsterdam, 1982).

${ }^{9}$ G. P. Morriss and P. A. Monson, Mol. Phys. 48, 181 (1983).

${ }^{10}$ E. Enciso, Mol. Phys. 60, 617 (1987); P. J. Rossky and W. T. D. Dale, J. Chem. Phys. 73, 2457 ( 1980 )

${ }^{11}$ O. Steinhauser and H. Bertagnolli, Chem. Phys. Lett. 78, 555 (1981).

${ }^{12}$ E. Lomba, M. Lombardero, and J. L. F. Abascal, J. Chem. Phys. 90, 7330 (1989).

${ }^{13}$ G. Stell, G. N. Patey, and J. S. Hoye, Adv. Chem. Phys. 48, 183 (1981).

${ }^{14}$ M. Neumann, Mol. Phys. 50, 841 (1983).

${ }^{15}$ A. Malijevsky and S. Labik, Mol. Phys. 60, 663 (1987).

${ }^{16}$ M. J. Gillan, Mol. Phys. 38, 1781 (1979).

${ }^{17}$ F. Lado, Comp. Phys. Comm. 39, 133 (1986); see also program DuMBELL from CPC Program Library, Queen's University of Belfast, N. Ireland.

${ }^{18}$ D. Levesque, G. N. Patey, and J. J. Weis, Mol. Phys. 34, 1077 (1977).

${ }^{19}$ G. N. Patey, D. Levesque, and J. J. Weis, Mol. Phys. 45, 733 (1982).

${ }^{20} \mathrm{G}$. Stell, in The Equilibrium Theory of Classical Fluids, edited by H. L. Frisch and J. L. Lebowitz (Benjamin, New York, 1964), p. II-171.

${ }^{21}$ S. M. Foiles, N. W. Ashcroft, and L. Reatto, J. Chem. Phys. 80, 4441 (1984). 